\title{
Efecto Mozart en el Aprendizaje Práctico de la Anatomía
}

\author{
The Mozart Effect in the Practical Learning of the Gross Anatomy \\ "Iván Claudio Suazo Galdames; "Mario Cantín López; "Guillermo Enrique Salgado Alarcón; "Carolina Haydée Gajardo \\ Contreras; "Paula Macarena Caballero Moyano; "Erick Gonzalo Trujillo Hernandez; *'Ivonne Patricia Bustos Alvarez \& \\ ***:Daniela Alejandra Zavando Matamala
}

SUAZO, G. I. C.; CANTín, L. M.; SAlgAdO, A. G. E.; GAJARDO, C. C. H.; CABALLERO, M. P. M.; TRUJILLO, H. E. G.; BUSTOS, A. I. P. \& ZAVANDO, M. D. A. Efecto Mozart en el aprendizaje práctico de la Anatomía. Int. J. Morphol., 26(3):713-717, 2008.

\begin{abstract}
RESUMEN: El término efecto Mozart comienza a utilizarse a partir de los trabajos de Rauscher et al. (1993), quienes observaron mejores resultados en tareas que requerían orientación temporo-espacial en estudiantes universitarios. El objetivo de este estudio es analizar el efecto que tiene en el aprendizaje práctico de la anatomía la aplicación de estímulos musicales relacionados con el denominado efecto Mozart. Participaron 254 estudiantes de las carreras de Odontología, Fonoaudiología y Tecnología Médica. Los grupos de estudio fueron sometidos durante toda una Unidad temática a estimulación auditiva utilizando el primer movimiento de la Sonata para dos Pianos en D Mayor (K. 448) de Mozart, los grupos controles desarrollaron su actividad práctica sin estimulación auditiva, al finalizar la unidad se realizó una evaluación práctica de reconocimiento de estructuras en el cadáver. Las medias de los resultados obtenidos fueron superiores en los sujetos sometidos a estimulación auditiva, estos resultados fueron estadísticamente significativos en las carreras de Odontología y Fonoaudiología, pero no en Tecnología Médica.
\end{abstract}

PALABRAS CLAVE: Efecto Mozart; Aprendizaje; Aprendizaje práctico; Anatomía.

\section{INTRODUCCIÓN}

El efecto de la música en el desarrollo de habilidades temporo-espaciales ha sido estudiado por numerosos autores (Rauscher et al., 1997; Gromko \& Poorman, 1998; Costa-Giomi, 1999; Bilhartz et al., 2000; Rauscher \& Zupan, 2000; Husain et al., 2002).

El término efecto Mozart comenzó a utilizarse a partir de los estudios de Rauscher et al. (1993), los cuales mostraron que alumnos universitarios obtuvieron mejores resultados en tareas que requerían orientación espacial, realizadas inmediatamente después de escuchar la Sonata para dos Pianos K. 448 en D Mayor de Mozart.

Este efecto ha sido discutido por algunos autores, quienes lo asocian a una preferencia musical (Cassity et al., 2007; McKelvie \& Low, 2002; Ho et al., 2003) o simplemente a un ejemplo de leyenda científica (Bangerter \& Heath, 2004).

En la enseñanza de la morfología se emplean diversas estrategias para que el alumno comprenda las relaciones espaciales que los elementos anatómicos presentan en el cuerpo humano. Inzunza et al. (1993), Guiraldes et al. (1995) e Inzunza \& Bravo (1999) se refirieron a la utilización de programas computacionales en la enseñanza de la anatomía macroscópica, determinando que eran métodos eficientes como apoyo para la comprensión de los contenidos prácticos, no obstante, no superaron al trabajo del alumno directo sobre el cadáver, conclusión similar a la que llegaron Rojas et al. (1999) en el campo de la histología, siendo un factor determinante la dificultad del estudiante para ubicarse espacialmente en la preparación anatómica con la cual está siendo evaluado. En este sentido los alumnos con un mayor desarrollo de su inteligencia espacial (Gardner, 1995) tendrían una mayor facilidad en la ubicación de estructuras tridimensionales. García-Hernández (2006) afirma que el aprendizaje práctico de la anatomía debe ser multifactorial, aprovechándose al máximo las diversas inteligencias que poseen los estudiantes.

El objetivo de este estudio es analizar el efecto que tiene en el aprendizaje práctico de la anatomía la aplicación de estímulos musicales relacionados con el denominado efecto Mozart.

\footnotetext{
* Unidad de Anatomía Normal, Facultad de Ciencias de la Salud, Universidad de Talca, Chile.

** Departamento de Salud Pública, Facultad de Ciencias de la Salud, Universidad de Talca, Chile.

****Departamento de Estomatología, Facultad de Ciencias de la Salud, Universidad de Talca, Chile.
} 


\section{SUJETOS Y MÉTODO}

En este estudio participaron alumnos de los cursos de Morfología y Anatomía Macroscópica de las carreras de Odontología ( $\mathrm{n}=103)$, Fonoaudiología $(\mathrm{n}=83)$ y Tecnología Médica $(\mathrm{n}=68)$ de la Facultad de Ciencias de la Salud de la Universidad de Talca, cohorte 2007. Cada uno de los cursos se encuentra dividido en al menos dos secciones el total de alumnos que participó en el estudio fue de 254, con una edad promedio de 18,7 años (DS 1,08) al momento de comenzar el estudio.

Para desarrollar las actividades prácticas de Anatomía, los cursos de Fonoaudiología y Tecnología Médica fueron divididos en dos secciones y el de Odontología se dividió en cuatro secciones. La fase de campo de este estudio se llevó a cabo durante dos unidades de los cursos respectivos (U1 y U2), en U1 una sección de Fonoaudiología y Tecnología Médica y dos secciones de Odontología fueron sometidas a estimulación, mientras que el resto de las secciones fue el grupo control. En U2 las secciones que fueron sometidas a estimulación auditiva correspondieron a los controles de U1.

Todas las secciones desarrollaron su actividad práctica de acuerdo a la metodología utilizada en la Unidad de Anatomía de la Universidad de Talca, un sistema estructurado basado en el sistema de mesas implementado por Rodríguez (1997) en la Universidad de los Andes, Chile. Cada alumno debe pasar por tres mesas, en cada una de las cuales, en un tiempo de 30 minutos debe reconocer los elementos indicados en la guía de trabajos prácticos que se entrega al comienzo del curso, pudiendo consultar a cualquiera de los 4 docentes que se encuentran en el laboratorio. Los contenidos de los cursos se estructuran en base a unidades temáticas cada una de las cuales presenta una evaluación teórica y otra práctica.

Los grupos de estudio fueron sometidos durante toda la Unidad temática a estimulación auditiva utilizando el primer movimiento de la Sonata para dos Pianos en D Mayor (K. 448) de Mozart, en disco compacto, con una duración de 8 min 29 segundos, en tres ocasiones, al comienzo de cada una de las mesas de trabajo.

La modalidad de reproducción fue la de sonido auditorio, a una intensidad de 60-70 dB descrito por Jausovec et al. (2006), utilizando un equipo reproductor de música y Subwoofer Fujitel 8800 W PMPO MOD. 7000, los alumnos se encontraron sentados en sus mesones de trabajo respectivos. Los estudiantes del grupo control desarrollaron la actividad práctica en las mismas condiciones que los del grupo de estudio, pero sin la estimulación auditiva descrita.
Al finalizar la unidad temática se realizó una evaluación práctica tipo gymkhana, descrita por Inzunza et al. (2003) en la cual se pidió a los alumnos identificar en preparaciones cadavéricas distintos elementos descritos por su guía de trabajos prácticos.

Los resultados de las evaluaciones prácticas fueron tabulados y se analizó mediante el programa SPSS para Windows 11.5. Se calcularon los estadísticos descriptivos y la significancia de las diferencias obtenidas fue calculada mediante la prueba $t$ para muestras independientes con un intervalo de confianza del $95 \%$.

\section{RESULTADOS}

El número de sesiones para cada carrera fue distinto, Odontología tuvo 6 sesiones de U1 y U2, Fonoaudiología tuvo 5 sesiones en U1 y 6 sesiones en U2 y Tecnología médica tuvo 3 sesiones en U1 y U2.

Las medias de los resultados obtenidos en la prueba práctica fueron superiores en los grupos sometidos a estimulación auditiva en las tres carreras participantes en este estudio y en todas las unidades. Los estadísticos descriptivos de los resultados obtenidos pueden observarse en la Tabla I.

Al comparar las medias obtenidas por unidad y por carrera, mediante la prueba t para muestras independientes, se obtuvieron resultados significativos en Odontología y en Fonoaudiología en U1 y U2, por lo que se rechaza la supuesta igualdad de medias entre estos grupos. En Tecnología Médica, en cambio, estos resultados no fueron significativos en ninguna de las unidades, por lo que no existe suficiente evidencia para rechazar la igualdad en las medias obtenidas. Los estadísticos de la prueba t se observan en la Tabla II.

\section{DISCUSIÓN}

Para Insunza \& Bravo el práctico de anatomía es indispensable para realizar una adecuada actividad de enseñanza-aprendizaje en la disciplina, por ello la ubicación tridimensional de estructuras anatómicas en el cadáver es uno de los primeros desafíos para el aprendizaje práctico de la anatomía. 
SUAZO, G. I. C.; CANTín, L. M.; SALGADO, A. G. E.; GAJARdO, C. C. H.; CABALLERO, M. P. M.; TRUJILlO, H. E. G.; BUSTOS, A. I. P. \& ZAVANDO, M. D. A. Efecto Mozart en el aprendizaje práctico de la Anatomía. Int. J. Morphol., 26(3):713-717, 2008.

Tabla I. Número de sesiones y estadísticos descriptivos de los grupos por unidad y carrera.

\begin{tabular}{lcccccc}
\hline & EFECTO & $\begin{array}{c}\text { Número de } \\
\text { sesiones }\end{array}$ & $\mathrm{N}$ & Media & Des viación típ. & $\begin{array}{c}\text { Error típ. De } \\
\text { la media }\end{array}$ \\
\hline U1ODO & si & 6 & 52 & 4.69202 & .748039 & .103734 \\
U2ODO & no & 6 & 51 & 4.02941 & .829046 & .116090 \\
& si & 6 & 51 & 3.85755 & .908371 & .127197 \\
U1FONO & no & 6 & 52 & 3.32115 & .954317 & .132340 \\
& si & 5 & 41 & 4.524229 & 1.0571154 & .1650937 \\
U2FONO & no & 5 & 42 & 3.497619 & 1.1105894 & .1713677 \\
& si & 4 & 42 & 4.45870 & .985881 & .155882 \\
U1TM & no & 4 & 41 & 3.70930 & 1.011085 & .154189 \\
& si & 3 & 35 & 4.8991 & .81867 & .13838 \\
& no & 3 & 33 & 4.5697 & .81336 & .14159 \\
& si & 3 & 33 & 4.379 & .8480 & .1499 \\
\hline
\end{tabular}

ODO: Odontología. FONO: Fonoaudiología. TM: Tecnología Médica.

Tabla II: Estadísticos prueba t para muestras independientes, no se ha asumido igualdad de varianzas.

\begin{tabular}{|c|c|c|c|c|c|c|c|}
\hline & \multirow[t]{2}{*}{$t$} & \multirow[t]{2}{*}{ gl } & \multirow[t]{2}{*}{$\begin{array}{c}\text { Sig. } \\
\text { (bilateral) }\end{array}$} & \multirow[t]{2}{*}{$\begin{array}{l}\text { Diferencia de } \\
\text { medias }\end{array}$} & \multirow[t]{2}{*}{$\begin{array}{l}\text { Error típ. de la } \\
\text { diferencia }\end{array}$} & \multicolumn{2}{|c|}{$\begin{array}{c}95 \% \text { Intervalo de confianza para la } \\
\text { diferencia }\end{array}$} \\
\hline & & & & & & Inferior & Superior \\
\hline U1ODO & 4.256 & 99.520 & .000 & .66261 & .155684 & .353716 & .971499 \\
\hline U2ODO & 2.922 & 100.911 & .004 & .53640 & .183557 & .172264 & .900526 \\
\hline U1FONO & 4.314 & 80.950 & .000 & 1.026610 & .2379555 & .5531490 & 1.5000714 \\
\hline U2FONO & 3.418 & 80.814 & .001 & .74940 & .219256 & .313131 & 1.185664 \\
\hline U1TM & 1.664 & 65.814 & .101 & .3294 & .19798 & -.06586 & .72475 \\
\hline U2TM & 2.330 & 65.623 & .063 & .490 & .2104 & .0702 & .9106 \\
\hline
\end{tabular}

Basándonos en estudios de Rauscher et al. (1993) acerca del efecto Mozart en estudiantes universitarios, analizamos el efecto de una sonata de Mozart en el rendimiento de grupos de estudiantes sometidos a estimulación, en una prueba práctica de ubicación de estructuras en preparaciones cadavéricas.

Los resultados mostraron que los alumnos sometidos a la estimulación auditiva presentaron una mayor capacidad para reconocer estructuras en preparaciones cadavéricas, lo que concuerda por lo comunicado por diversos autores (Graziano et al. 1999; Costa-Giomi, 2000; Hetland, 2000a, 2000b), estos resultados fueron significativos para las carreras de Odontología y Fonoaudiología, pero no para la carrera de Tecnología Médica, este hecho puede relacionarse con el menor número de sesiones prácticas en las cuales fueron sometidos a estimulación auditiva. La relación entre las oportunidades de exposición y el desarrollo del efecto Mozart fue reportado por Rauscher $(2000,2003)$ y Jausovec et al. 
La aparente mejora en la capacidad de orientación espacial permite al estudiante generar aprendizajes prácticos significativos lo que se relaciona con un mejor rendimiento en las evaluaciones prácticas. Rauscher et al. (1993), señalan que el denominado efecto Mozart está presente sólo durante los primeros 10 minutos luego de la exposición, por lo que en esta experiencia resultaría eficiente durante el paso práctico como ayuda en el aprendizaje, para Lewis (2002) en cambio la estimulación auditiva tiene resultados a largo plazo ya que mejora la capacidad de retención de los contenidos, lo que favorecería su evocación al momento de la evaluación práctica.

Aunque estos resultados señalan la presencia de efecto Mozart en algunas de los grupos analizados, en el aprendizaje práctico de la anatomía el énfasis debe ponerse en la necesidad que éste se desarrolle sobre material cadavérico.

SUAZO, G. I. C.; CANTín, L. M.; SAlgAdO, A. G. E.; GAJARDO, C. C. H.; CABALLERO, M. P. C.; TRUJILLO, H. E. G.; BUSTOS, A. I. P. \& ZAVANDO, M. D. A. The Mozart effect in the practical learning of the gross anatomy. Int. J. Morphol., 26(3):713717, 2008.

SUMMARY: The term Mozart effect begins to be used starting from the works of Rauscher et al. (1993) who observed better results in tasks that required temporo-space orientation in university students. The objective of this study is to analyze the effect that has in the practical learning of the anatomy the application of musical stimuli related with the denominated Mozart effect. This study involved 254 participants of the careers of Dentistry, Fonoaudiology and Medical Technology. The study groups were subjected during an entire thematic Unit to auditory stimulation using the first movement of the Mozart's Sonata for Two Pianos K.448, the groups controls developed their practical activity without auditory stimulation, when concluding the unit they was carried out a practical evaluation of recognition of structures in the cadaver. The mean of the obtained results were superior in the subjected fellows to auditory stimulation, these results were statistically significant in the careers of Dentistry and Fonoaudiology, but not in Medical Technology.

KEY WORDS: Mozart effect; Learning; Practical learning; Anatomy.

\section{REFERENCIAS BIBLIOGRÁFICAS}

Cassity, H. D.; Henley, T. B. \& Markley, R. P. The Mozart effect: musical henomenon or musical preference? A more ecologically valid reconsideration. J. of Instructional Psychology, 34(1):13-7, 2007.

Bangerter, A. \& Heath, C. The Mozart effect: Tracking the evolution of a scientific legend. Br. J. Soc. Psychol., 43:605-23, 2004.

Bilhartz, T. D.; Bruhn, R. A. \& Olson, J. E.. The effect of early music training on child cognitive development. $J$. Appl. Dev. Psychol., 20(4):615-36, 2000.

Costa-Giomi, E. The effects of three years of piano instruction on children's cognitive development. $J$. of Research in Music Education, 47(3):198-212, 1999.

Costa-Giomi, E. The relationship between absolute pitch and spatial abilities. In: Woods, C.; Luck, G.; Brochard, R.; Seddon, F. \& Sloboda, J. A. Eds., Proceedings of the sixth international conference on music perception and cognition. Keele, Keele University, Department of Psychology, 2000.

García-Hernández, F. Evaluación práctica de la Anatomía basada en la teoría de las inteligencias múltiples de Gardner, Carrera de Odontología de la Universidad de Antofagasta, Chile. Int. J. Morphol., 24(1):83-8, 2006.

Gardner, H. Estructuras de la mente. La teoría de las inteligencias múltiples. $2^{\circ}$ ed. México, Fondo de Cultura Económica, 1995.

Graziano, A. B.; Peterson, M. \& Shaw, G. L. Enhanced learning of proportional math through music training and spatial-temporal training. Neurol. Res., 21(2):139-52, 1999.

Gromko, J. E. \& Poorman, A. S. The effect of music training on preschoolers' spatial-temporal task performance. $J$. of Research in Music Education, 46(2):173-81, 1998.

Guiraldes, H.; Oddó, H.; Ortega, X. \& Oyarzo, M. Métodos computacionales y gráficos de apoyo al aprendizaje de la Anatomía Humana. Rev. Chil. Anat., 13(1):67-71, 1995.

Hetland, L. Listening to music enhances spatial-temporal reasoning: Evidence for the Mozart Effect. J. Aesthetic Educ., 34(3-4):105-48, 2000a. 
Hetland, L. Learning to make music enhances spatial reasoning. J. of Aesthetic Education, 34(3-4):179-238, 2000b.

Ho, Y. C.; Cheung, M. C. \& Chan, A. S. Music training improves verbal but not visual memory: cross-sectional and longitudinal explorations in children. Neuropsychology, 17(3):439-50, 2003.

Husain, G.; Thompson, W. F. \& Schellenberg, E. G. Effects of musical tempo and mode on arousal, mood, and spatial abilities. Music Percept., 20(2):151-71, 2002.

Inzunza, O.; Bravo, H.; Garcia-Huidobro, C.; Contreras, P.; Riquelme, S. \& Valenzuela, A. Software docente para la enseñanza en Anatomía. Rev. Chil. Anat., 11:81, 1993.

Inzunza, O. \& Bravo, H. Impacto de dos programas computacionales de Anatomía Humana en el rendimiento del conocimiento práctico de los alumnos. Rev. Chil. Anat., 17(2):205-9, 1999.

Inzunza, O.; D'Acuna, E. \& Bravo, H. Evaluación práctica de anatomía. Rendimiento de los alumnos de primer año de medicina ante distintas formas de preguntar. Int. J. Morphol., 21(2):131-6, 2003.

Jausovec, N.; Jausovec, K. \& Gerlic, I. The influence of Mozart's music on brain activity in the process of learning. Clin. Neurophysiol., 117(12): 2703-14, 2006.

Lewis, E. The relationship of listening to classical music on first grades'Ability to retain information. Master of Arts Actions Research Project, Johnson Bible College, Tenneessee. ERIC, 2002.

McKelvie, P. \& Low, J. Listening to Mozart does not improve children's spatial ability: Final curtains for the Mozart effect. Br. J. Devel. Psych. 20(2):241-58, 2002.

Rauscher, F. H.; Shaw, G. L. \& Ky, K. N. Music and spatial task performance. Nature, 365(6447):611, 1993.

Rauscher, F. H.; Shaw, G. L.; Levine, L. J.; Wright, E. L.; Dennis, W. R. \& Newcomb, R. L. Music training causes long-term enhancement of preschool children's spatialtemporal reasoning. Neurol. Res., 19(1):1-8, 1997.

Rauscher, F. H. \& Zupan, M. Classroom keyboard instruction improves kindergarten children's spatial-temporal performance: A field experiment. Early Childhood Res. Q., 15(2):215-28. 2000
Rauscher, F. H. \& LeMieux, M. T. Piano, rhythm, and singing instruction improve different aspects of Spatial-Temporal reasoning in head start children. Poster presented at the annual meeting of the Cognitive Neuroscience Society, New York, 2003.

Rodríguez, T. A. Estrategias de enseñanza y aprendizaje en anatomía normal, Memoria de investigación y difusión científica. Universidad de los Andes, Santiago-Chile, 1997.

Rojas, M.; Montiel, E.; Montiel, J.; Ondarza, A. \& Rodríguez, H. Estudio comparado entre métodos de enseñanza tradicional y computacional en Histología. Rev. Chil. Anat., 17(1):81-5, 1999.

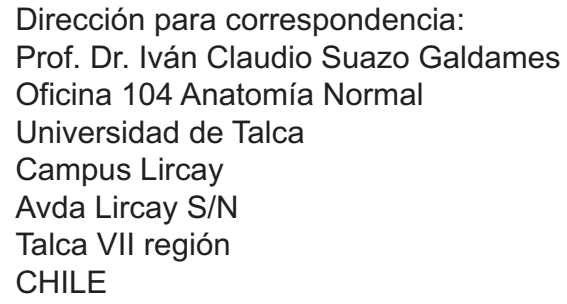

Recibido : 03-09-2007

Aceptado: 22-06-2208 